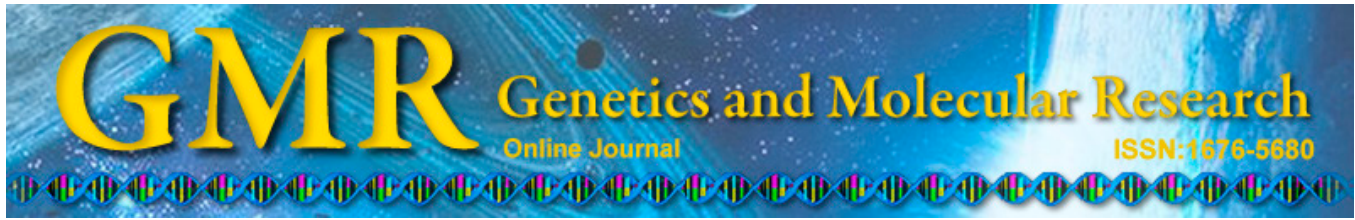

\title{
Association between the hsa-miR-146a rs2910164 functional polymorphism with susceptibility to intracranial aneurysm
}

\author{
X.T. Sima, J.G. Xu, J. Li and C. You \\ Department of Neurosurgery, West China Hospital of Sichuan University, \\ Chengdu, Sichuan, China \\ Corresponding author: C. You \\ E-mail: youchao1977@163.com
}

Genet. Mol. Res. 14 (3): 7680-7686 (2015)

Received September 17, 2014

Accepted April 6, 2015

Published July 13, 2015

DOI http://dx.doi.org/10.4238/2015.July.13.13

\begin{abstract}
Vascular inflammation has been shown to be involved in the pathogenesis of intracranial aneurysms (IA). MiRNAs are key molecules that participate in the regulation of many important biological processes including inflammation. Studies on the $h s a-m i R-146 a$ rs2910164 polymorphism and its association with different inflammatory related diseases have engendered inconsistent results, and until now, there have been no reports on the association between this polymorphism and the susceptibility to IA. In this study, we aimed to investigate whether the rs2910164 polymorphism is involved in the process of IA. We genotyped 164 patients with IA and 478 healthy controls using a polymerase chain reaction-restriction fragment length polymorphism assay. All subjects were Chinese. The distributions of rs2910164 genotypes and alleles between patients with IA and healthy controls were similar $[\mathrm{CG}$ vs $\mathrm{CC}$ : odds ratio $(\mathrm{OR})=$ $0.701,95 \%$ confidence interval $(\mathrm{CI})=0.456-1.080 ; \mathrm{GG}$ vs $\mathrm{CC}$ : $\mathrm{OR}=0.920,95 \% \mathrm{CI}=0.524-1.617 ; \mathrm{G} v s \mathrm{C}: \mathrm{OR}=0.939,95 \% \mathrm{CI}=$ 0.731-1.208, respectively]. No association was found between the
\end{abstract}


hsa-miR-146a rs2910164 polymorphism and the risk of IA in the analyzed population.

Key words: Intracranial aneurysm; hsa-miR-146a; Polymorphism; Susceptibility

\section{INTRODUCTION}

Despite its high morbidity, approximately $5 \%$ of the general population carry an undiagnosed unruptured intracranial aneurysm (Iwamoto et al., 1999). Because of the catastrophic consequences of their rupture, nearly half of patients die during the first post-rupture month; $60 \%$ of deaths occur within two days of the onset of subarachnoid hemorrhage (SAH) (Sarti et al., 1991; Broderick et al., 1994). Intracranial aneurysms (IA) are also becoming a major ongoing public health problem because half of the survivors require long-term care (Hackett and Anderson, 2000). Therefore, it is of great value to better understand the underlying mechanisms of IA to facilitate its prevention and treatment. Conclusions drawn from studies on samples from patients with IA have strongly suggested that chronic inflammation is involved in the pathogenesis of this condition (Chyatte et al., 1999; Jayaraman et al., 2005), and further evidence has been provided from various experimental findings from rodent IA models (Aoki et al., 2007, 2009; Aoki and Nishimura, 2010). Infiltration of macrophages and T cells (Chyatte et al., 1999), activation and deposition of complement or immunoglobulin (Chyatte et al., 1999; Tulamo et al., 2006), and cell apoptosis (Pentimalli et al., 2004; Guo et al., 2007) have been detected in IA walls at the histological level, further supporting the role of active involvement of inflammation in IA formation.

MiRNAs are molecules that participate in diverse biological processes, most commonly as negative regulators at the level of posttranscriptional repression. Hsa-miR-146a was first identified as an immune system regulator by Taganov et al. (2006). Promoter analysis of the $h s a-m i R-146 a$ gene revealed that the induction of $h s a-m i R-146 a$ transcription in response to lipopolysaccharide, tumor necrosis factor- $\alpha$ (TNF- $\alpha$ ), and/or interleukin-1 beta (IL-1 $\beta$ ) is

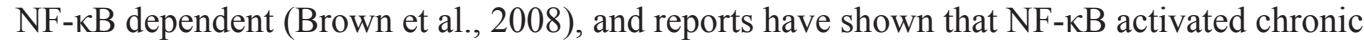
inflammation is associated with increased formation of IA (Fukuda et al., 2000; Sadamasa et al., 2003). Further studies characterizing $h s a-m i R-146 a$ expression have revealed a negative regulatory loop, in which NF- $\mathrm{kB}$ activation upregulates the $h s a-m i R-146 a$ gene, downregulates its direct targets (tumor necrosis factor receptor-associated family members and interleukin-1 receptor-associated kinase), and in turn reduces the activity of NF- $\mathrm{B}$ (Taganov, 2006).

The rs2910164 polymorphism in the stem structure of the $h s a-m i R-146 a$ precursor have been shown to affect the mature expression levels of hsa-miR-146a (Jazdzewski et al., 2008, 2009; Xu et al., 2010; Yue et al., 2011). Therefore, we hypothesized that the rs2910164 polymorphism might participate in the process of IA formation or rupture.

\section{MATERIAL AND METHODS}

\section{Study population}

Detailed information population used in this study has been described previously 
(Sima et al., 2013). Briefly, 642 subjects including 164 patients with IA and 478 controls participated in this hospital-based case-control study. Subjects were recruited between January 2008 and September 2009 from the West China Hospital. All subjects were genetically unrelated ethic Han Chinese and lived in the Sichuan Province of southwest China. No significant difference existed between patients and controls in age distribution. There were two kinds of patients with IA: ruptured and un-ruptured. The former were diagnosed because of the rupture of IA and the un-ruptured were diagnosed by digital subtraction angiography (DSA). The control group was enrolled from the physical examination center of the same hospital during the same time period, and those who had diseases of the nervous system or other severe diseases were excluded. The study was approved by the ethics committee of the West China Hospital, and written informed consent was obtained from all participants or their parent/carer.

\section{Genotyping}

Approximately $2 \mathrm{~mL}$ venous blood was collected in an ethylenediaminetetraacetic acid (EDTA)-anticoagulation tube. Genomic DNA was extracted from blood samples using a commercial extraction kit (Bioteke Corporation, Beijing, China). hsa-miR-146a rs2910164 genotypes were determined using a polymerase chain reaction-restriction fragment length polymorphism (PCR-RFLP) assay, and confirmed by DNA sequencing; the results were $100 \%$ consistent. The gel images were read by two investigators. The primers, reaction conditions, and the enzyme used were as described by $\mathrm{Hu}$ et al. (2008).

\section{Statistical analysis}

The genotype and allele frequencies of the rs2910164 polymorphism were calculated with Modified-Powerstates standard edition software. Hardy-Weinberg equilibrium for the control group was tested with a chi-square test. Genotype and allele frequencies of the rs2910164 polymorphism were compared between patients and controls using chisquare tests. Odds ratios (ORs) and 95\% confidence intervals (CIs) were used to assess the strength of association conferred by a particular allele or genotype. $\mathrm{P}<0.05$ was considered to be statistically significant and all statistical tests were two sided. All the data analyses were performed using the SPSS for Windows software package version 19.0 (SPSS Inc., Chicago, IL, USA).

\section{RESULTS}

The characteristics of patients and controls are presented in Table 1. There were more females among the patients (63.4\%) compared to the controls (57.1\%). The genotype and allele frequencies of rs2910164 are shown in Table 2. The genotype distribution in the control group conformed to Hardy-Weinberg equilibrium. The hsa-miR-146a rs2910164 polymorphism showed no statistical differences between patients with IA and controls for either genotypic or allelic distributions $(\mathrm{CG} v s \mathrm{CC}$ : $\mathrm{OR}=0.701,95 \% \mathrm{CI}=0.456-1.080$; $\mathrm{GG}$ vs $\mathrm{CC}: \mathrm{OR}=0.920,95 \% \mathrm{CI}=0.524-1.617 ; \mathrm{G}$ vs $\mathrm{C}: \mathrm{OR}=0.939,95 \% \mathrm{CI}=0.731-1.208$, respectively). 
Table 1. Demographics of the patients with intracranial aneurysm and controls.

\begin{tabular}{lcc}
\hline Variables & Controls, $\mathrm{N}=478(\%)$ & Patients with IA, N = 164 (\%) \\
\hline Age $(\mathrm{y})$ & $57.6( \pm 8.9)$ & $53.1( \pm 13.1)$ \\
Gender & $205(42.9)$ & $60(36.6)$ \\
$\quad$ Male & $273(57.1)$ & $104(63.4)$ \\
Female & - & 142 \\
Number of aneurysms & - & 22 \\
$\quad 1$ &
\end{tabular}

Table 2. Genotype and allele frequencies of the rs2910164 in the controls and patients with IA.

\begin{tabular}{lrrcr}
\hline Rs2910164 & Controls (\%) & IA (\%) & OR (95\%CI) & P value \\
\hline Genotypes & & & & \\
CC & $134(28.0)$ & $37(22.5)$ & 1 (Reference) & 0.106 \\
CG & $254(53.2)$ & $100(61.0)$ & $0.701(0.456-1.080)$ & 0.773 \\
GG & $90(18.8)$ & $27(16.5)$ & $0.920(0.524-1.617)$ & \\
Alleles & $522(54.6)$ & $174(53.0)$ & 1 (Reference) & 0.626 \\
C & $434(45.4)$ & $154(47.0)$ & $0.939(0.731-1.208)$ & \\
G & & & & \\
\hline
\end{tabular}

$\mathrm{IA}=$ intracranial aneurysm.

\section{DISCUSSION}

In this study, we investigated the association between the common rs2910164 polymorphism in the mature hsa-miRNA-146a and the risk of IA in a Chinese population. For the first time, we found that neither genotype nor allele distributions were significantly different between patients and controls. These results suggested that the rs2910164 variant in the mature hsa-miRNA-146a might not be involved in the process of IA.

There are two members in hsa-miR-146 family: hsa-miR-146a and hsa-miR-146b, the genes for which are located on chromosomes 5 and 10, respectively. Hsa-miRNA-146a is transcribed from a precursor gene (pre-miRNA-146a), and upregulation of hsa-miR-146a is related to macrophage inflammation (Taganov et al., 2006, 2007; O'Connell et al., 2010). The infiltration of monocytes and macrophages is a characteristic of inflammation in the vessel wall and plays a causal role in arterial remodeling, contributing to the formation of aneurysms (Schober and Weber, 2005). Previously, a G $>$ C nucleotide substitution in the stem structure of the mature hsa-miR-146a (rs2910164) was reported to reduce the expression level of the mature miRNA (Jazdzewski et al., 2008). To date, the hsa-miR-146a rs2910164 polymorphism has been primarily investigated for its association with several inflammation or immune-associated diseases. For instance, there have been reports that the hsa-miR-146a rs2910164 polymorphism was positively associated with asthma (Jiménez-Morales et al., 2012) and systemic sclerosis (Sakoguchi et al., 2013). Nevertheless, negative results have been found for this variant in systemic lupus erythematosus, rheumatoid arthritis, and moyamoya disease (Gao et al., 2010; Zhang et al., 2010; Löfgren et al., 2012; Park et al., 2012).

Although the exact role of miRNA genetic variants in IA remains unknown, there have been many related reports suggesting potential mechanism. Recently, Jayaraman et al. (2005) have reported that the pro-inflammatory cytokine TNF- $\alpha$ was highly expressed in IA 
walls and that the apoptotic pathway regulated by TNF- $\alpha$ was involved in the progression of aneurysm development. Further evidence has also supported the view that apoptosis occurs in human IA walls (Sakaki et al., 1997; Hara et al., 1998; Pentimalli et al., 2004). In experimental studies, the inflammatory cytokine IL- $1 \beta$ induced apoptosis in the medial smooth muscle cells of IA walls, and deficiency of IL- $1 \beta$ resulted in the decreased incidence of IA formation (Sadamasa et al., 2003; Moriwaki et al., 2006). Furthermore, TNF- $\alpha$ and IL-1 $\beta$ have been shown to downregulate pro-collagen biosynthesis in various types of cells at the transcriptional level (Mauviel et al., 1991; Keen et al., 1994). In addition, activation of NF- $\mathrm{kB}$ has been associated with downregulation of the pro-collagen $\alpha 1$ gene, and shown to eventually contribute to IA progression (Rippe et al., 1999; Roebuck et al., 2001). In 2006, the Baltimore laboratory reported that the induction of $h s a-m i R 146 a$ transcription is NF- $\mathrm{kB}$ dependent, and in turn, that hsa-miR-146a potentially downregulates TRAF6 and IRAK to provide a brake on inflammation, implicating its negative regulatory role in fine-tuning the immune response (Taganov et al., 2006). We therefore hypothesized that the negative regulatory function of has-miR-146a in the inflammatory pathway might play an important role in the pathogenesis of IA. However, in this study we failed to find any association between the hsa-miR-146a rs2910164 polymorphism and IA risk. It is important to conduct further large, multi-center studies to assess the role of the rs2910164 polymorphism in the formation of IA in different ethnic groups.

There were some limitations in our study. First, the statistical power was limited due to the moderately small number of patients. Second, all patients were selected from the same hospital during one time period, and thus, might have been unrepresentative of patients with IA in the general population. Third, the lack of data on $h s a-m i R-146 a$ expression levels in IA limited our further analysis of the association between rs2910164 variation and hsa-miR-146a expression.

In conclusion, this study investigated the association between the rs 2910164 polymorphism and IA susceptibility; no association was found between the rs2910164 genotype and IA risk. Future studies with larger samples are needed to investigate the role of hsa-miR-146a in IA, which will be helpful for further elucidation of one of the potential causes of IA.

\section{Conflicts of interest}

The authors declare no conflict of interest.

\section{ACKNOWLEDGMENTS}

Research supported by the Project of the National Science \& Technology Pillar Program (\#2011BAI08B05).

\section{REFERENCES}

Aoki T and Nishimura M (2010). Targeting chronic inflammation in cerebral aneurysms: focusing on NF-kappa B as a putative target of medical therapy. Expert Opin. Ther. Tar. 14: 265-273.

Aoki T, Kataoka H, Shimamura M, Nakagami H, et al. (2007). NF-kB Is a key mediator of cerebral aneurysm formation. Circulation 116: 2830-2840.

Aoki T, Kataoka H, Ishibashi R, Nozaki K, et al. (2009). Impact of monocyte chemoattractant protein-1 deficiency on cerebral aneurysm formation. Stroke 40: 942-951.

Broderick JP, Brott TG, Duldner JE, Tomsick T, et al. (1994). Initial and recurrent bleeding are the major causes of death following subarachnoid hemorrhage. Stroke 25: 1342-1347. 
Brown KD, Claudio E and Siebenlist U (2008). The roles of the classical and alternative nuclear factor-kappa B pathways: potential implications for autoimmunity and rheumatoid arthritis. Arthritis Res. Ther. 10: 212.

Chyatte D, Bruno G, Desai S and Todor DR (1999). Inflammation and intracranial aneurysms. Neurosurgery 45: 11371146; discussion 1146-1147.

Fukuda S, Hashimoto N, Naritomi H, Nagata I, et al. (2000). Prevention of rat cerebral aneurysm formation by inhibition of nitric oxide synthase. Circulation 101: 2532-2538.

Gao LB, Bai P, Pan XM, Jia J, et al. (2010). The association between two polymorphisms in pre-miRNAs and breast cancer risk: a meta-analysis. Breast Cancer Res. Treat. 125: 571-574.

Guo F, Li Z, Song L, Han T, et al. (2007). Increased apoptosis and cysteinyl aspartate specific protease-3 gene expression in human intracranial aneurysm. J. Clin. Neurosci. 14: 550-555.

Hackett ML and Anderson CS (2000). Health outcomes 1 year after subarachnoid hemorrhage - An international population-based study. Neurology 55: 658-662.

Hara A, Yoshimi N and Mori H (1998). Evidence for apoptosis in human intracranial aneurysms. Neurol. Res. 20: 127-130.

Hu Z, Chen J, Tian T, Zhou X, et al. (2008). Genetic variants of miRNA sequences and non-small cell lung cancer survival. J. Clin. Invest. 118: 2600-2608.

Iwamoto H, Kiyohara Y, Fujishima M, Kato I, et al. (1999). Prevalence of intracranial saccular aneurysms in a Japanese community based on a consecutive autopsy series during a 30-year observation period. The Hisayama study. Stroke 30: 1390-1395.

Jayaraman T, Berenstein V, Li X, Mayer J, et al. (2005). Tumor necrosis factor alpha is a key modulator of inflammation in cerebral aneurysms. Neurosurgery 57: 558-564.

Jazdzewski K, Murray EL, Franssila K, Jarzab B, et al. (2008). Common SNP in pre-miR-146a decreases mature miR expression and predisposes to papillary thyroid carcinoma. Proc. Natl. Acad. Sci. U. S. A. 105: 7269-7274.

Jazdzewski K, Liyanarachchi S, Swierniak M, Pachucki J, et al. (2009). Polymorphic mature microRNAs from passenger strand of pre-miR-146a contribute to thyroid cancer. Proc. Natl. Acad. Sci. U. S. A. 106: 1502-1505.

Jiménez-Morales S, Gamboa-Becerra R, Baca V, Del Rio-Navarro BE, et al. (2012). MiR-146a polymorphism is associated with asthma but not with systemic lupus erythematosus and juvenile rheumatoid arthritis in Mexican patients. Tissue Antigens 80: 317-321.

Keen RR, Nolan KD, Cipollone M, Scott E, et al. (1994). Interleukin-1 beta induces differential gene expression in aortic smooth muscle cells. J. Vasc. Surg. 20: 774-784; discussion 784-786.

Löfgren SE, Frostegård J, Truedsson L, Pons-Estel BA, et al. (2012). Genetic association of miRNA-146a with systemic lupus erythematosus in Europeans through decreased expression of the gene. Genes Immun. 13: 268-274.

Mauviel A, Heino J, Kähäri VM, Hartmann DJ, et al. (1991). Comparative effects of interleukin-1 and tumor necrosis factor-alpha on collagen production and corresponding procollagen mRNA levels in human dermal fibroblasts. $J$. Invest. Dermatol. 96: 243-249.

Moriwaki T, Takagi Y, Sadamasa N, Aoki T, et al. (2006). Impaired progression of cerebral aneurysms in interleukin-1 beta-deficient mice. Stroke 37: 900-905.

O'Connell RM, Rao DS, Chaudhuri AA and Baltimore D (2010). Physiological and pathological roles for microRNAs in the immune system. Nat. Rev. Immunol. 10: 111-122.

Park YS, Jeon YJ, Lee BE, Kim TG, et al. (2012). Association of the miR-146aC $>$ G, miR-196a2C $>$ T, and miR-499A $>$ G polymorphisms with moyamoya disease in the Korean population. Neurosci. Lett. 521: 71-75.

Pentimalli L, Modesti A, Vignati A, Marchese E, et al. (2004). Role of apoptosis in intracranial aneurysm rupture. $J$. Neurosurg. 101: 1018-1025.

Rippe RA, Schrum LW, Stefanovic B, Solis-Herruzo JA, et al. (1999). NF-kappaB inhibits expression of the alpha1(I) collagen gene. DNA Cell Biol. 18: 751-761.

Roebuck KA, Vermes C, Carpenter LR, Fritz EA, et al. (2001). Down-regulation of procollagen alpha1[I] messenger RNA by titanium particles correlates with nuclear factor kappaB (NF-kappaB) activation and increased rel A and NF-kappaB1 binding to the collagen promoter. J. Bone Miner. Res. 16: 501-510.

Sadamasa N, Nozaki K and Hashimoto N (2003). Disruption of gene for inducible nitric oxide synthase reduces progression of cerebral aneurysms. Stroke 34: 2980-2984.

Sakaki T, Kohmura E, Kishiguchi T, Yuguchi T, et al. (1997). Loss and apoptosis of smooth muscle cells in intracranial aneurysms. Studies with in situ DNA end labeling and antibody against single-stranded DNA. Acta Neurochir. 139: 469-474; discussion 474-475.

Sakoguchi A, Jinnin M, Makino T, Kajihara I, et al. (2013). The miR-146a rs2910164 C/G polymorphism is associated with telangiectasia in systemic sclerosis. Clin. Exp. Dermatol. 38: 99-100.

Sarti C, Tuomilehto J, Salomaa V, Sivenius J, et al. (1991). Epidemiology of subarachnoid hemorrhage in Finland from 1983 to 1985. Stroke 22: 848-853. 
Schober A and Weber C (2005). Mechanisms of monocyte recruitment in vascular repair after injury. Antioxid. Redox Signal. 7: 1249-1257.

Sima X, Xu J, Li J and You C (2013). Association between NFKB1 -94 insertion/deletion ATTG polymorphism and risk of intracranial aneurysm. Genet. Test. Mol. Biomarkers 17: 620-624.

Taganov KD, Boldin MP, Chang KJ and Baltimore D (2006). NF-אB-dependent induction of microRNA miR-146, an inhibitor targeted to signaling proteins of innate immune responses. Proc. Natl. Acad. Sci. U. S. A. 103: 12481-12486.

Taganov KD, Boldin MP and Baltimore D (2007). MicroRNAs and immunity: tiny players in a big field. Immunity 26: 133-137.

Tulamo R, Frösen J, Junnikkala S, Paetau A, et al. (2006). Complement activation associates with saccular cerebral artery aneurysm wall degeneration and rupture. Neurosurgery 59: 1069-1076, discussion 1076-1077.

Xu B, Feng NH, Li PC, Tao J, et al. (2010). A functional polymorphism in Pre-miR-146a gene is associated with prostate cancer risk and mature miR-146a expression in vivo. Prostate 70: 467-472.

Yue C, Wang M, Ding B, Wang W, et al. (2011). Polymorphism of the pre-miR-146a is associated with risk of cervical cancer in a Chinese population. Gynecologic Oncol. 122: 33-37.

Zhang J, Yang B, Ying B, Li D, et al. (2010). Association of pre-microRNAs genetic variants with susceptibility in systemic lupus erythematosus. Mol. Biol. Rep. 38: 1463-1468. 